\title{
Factors associated with community-acquired urinary tract infections among adults attending assessment centre, Mulago Hospital Uganda.
}

\author{
Deus Kabugo ${ }^{1}$, Samuel Kizito ${ }^{4,5}$, Dave Dhara Ashok ${ }^{1}$, Kiwanuka Alexander Graham ${ }^{1}$, Nabimba Ronald ${ }^{1}$, \\ Namunana Sandra ${ }^{1}$, Kabaka M Richard ${ }^{2}$, Beatrice Achan ${ }^{3}$, Florence C Najjuka ${ }^{3}$.
}

1. Makerere University College of health sciences, Kampala

2. School of veterinary medicine, Makerere University, Kampala

3. Department of Medical Microbiology, Makerere University, Kampala

4. Clinical Epidemiology Unit, Makerere University College of Health sciences

5. Uganda Tuberculosis Surveillance Projects, Makerere University College of Health Sciences

\begin{abstract}
Background: Urinary tract infections (UTI) are a common medical problem affecting the general population and thus commonly encountered in medical practice, with the global burden of UTIs at about 150 million people. Because uropathogens largely originate from colonic flora, they are easy to predict, and this is the rationale for empirical treatment in Community Acquired-UTI (CA-UTIs). With the increasing prevalence of drug-resistant bacteria among adults with CA-UTI in Uganda, it is no longer adequate to manage CA-UTIs on empiric regimen without revising the susceptibility patterns of common CA-UTI causative agents. Thus in this study we set out to identify: The factors associated with CA-UTIs, the common uropathogens and the drug sensitivity patterns of the common uropathogens cultured.

Methodology: This was a cross-sectional study that was conducted in adults who presented with symptoms of a UTI at Mulago Hospital, assessment center. There were 139 patients who consented to the study and were recruited, an interviewer administered questionnaire was used to collect information from the study participants as regards demographic, social and clinical characteristics and Mid Stream Urine (MSU) samples were collected for urinalysis, culture and antibiotic susceptibility testing using the Kirby-Bauer disc diffusion technique was applied to the isolates.Numeric data were summarized using measures of central tendency while the categorical data was summarized using proportions and percentages.

Results: Age, female sex and marital status were factors that were significantly associated with CA-UTIs. Fifty four (54) cultures were positive for UTI with 26 giving pure growths. The commonest uropathogen isolated was Escherichia coli at 50\%, this was followed by Staphylococcus aureus at 15.4\%. The sensitivity of Escherichia coli to Ampicillin and Nitrofurantoin were $78.6 \%, 64.3 \%$ respectively, and the sensitivity of Staphylococcus aureus to ciprofloxacin, Nitrofurantoin and gentamycin were 100\%, 66.7\% and $66.7 \%$ respectively.

Conclusion: There are known factors associated with CA-UTIs such as age, female sex. There was generally high sensitivity to nitrofurantoin and gentamycin by most of the uropathogens isolated, and high resistance to the common antibiotics such as nalidixic acid and erythromycin thus a need for a bigger study that can be used to effect the change of the current recommendations in the Uganda Clinical Guidelines as regards empirical management of CA-UTIs.
\end{abstract}

Keywords: Community-acquired urinary tract infections, assessment centre, Mulago Hospital Uganda.

DOI: http://dx.doi.org/10.4314/ahs.v16i4.31

Cite as: Kabugo D, Kizito S, Ashok DD, Graham AK, Nabimba R, Namunana S, Kabaka MR, Achan B, Najjuka FC. Factors associated with community-acquired urinary tract infections among adults attending assessment centre, Mulago hospital Uganda. Afri Health Sci2016;16(4): 1131-1142. http:/ / dx.doi.org/10.4314/abs.v16it.31

\section{Background}

Urinary tract infections (UTI) are a common medical problem affecting the general population and thus com-
Corresponding author:
Deus Kabugo,
Makerere University College
of Health Sciences,
Email: deusi2007@yahoo.com

monly encountered in medical practice. Community acquired urinary tract infection (CA-UTI); defined as an infection of the urinary tract that occurs in the community or within less than 48 hours of hospital admission and was not incubating at the time of hospital admission, is the second commonest diagnosed infection in the community ${ }^{1}$. The global burden of UTIs is about 150 million people ${ }^{2}$. Host associated and bacterial virulence factors are necessary in the pathogenesis of $\mathrm{UTI}^{3}$. The commonest organisms isolated in most CA-UTIs are Escherichia 
coli and Klebsiella $s p p^{4}$,Other bacteria isolated from UTI include the Enterococcus spp, Proteus spp, Pseudomonas aeruginosa and Staphylococci among others ${ }^{4-7}$. Because uropathogens largely originate from colonic flora, they are easy to predict. This is the rationale for empirical treatment in CAUTI.

The management of CA-UTI entails the prompt use of antibiotics to eliminate the pathogen to avoid complications including but not limited to scarring that could lead to hypertension and end-stage renal disease ${ }^{3}$. The antimicrobial susceptibility patterns of the organisms causing CA-UTI however, vary according to each region and from time to time due to the antibiotic prescription practices of health care workers (HCWs). It is thus mandatory to document the risk factors of CA-UTIs, microbes and antimicrobial susceptibility patterns of pathogens causing CA-UTI. The guidelines for empirical management of CA-UTIs in Uganda has been in place for a while yet the susceptibility patterns of the organisms have been demonstrated to change ${ }^{8,9}$. With the increasing prevalence of drug-resistant bacteria among adults with CA-UTI in Uganda, it is no longer adequate to manage CA-UTIs on empiric regimen without revising the susceptibility patterns of common CA-UTI causative agents.

The empirical treatment limits opportunities for surveillance of antibiotic resistance among pathogens that cause CA-UTI, and also if regularly updated to match changing susceptibility patterns; empirical treatment is a convenient strategy for effective resource utilization. In this study we set out to identify 1) The factors associated with CA-UTIs, 2) The common uropathogens and 3) The drug sensitivity patterns of the common uropathogens cultured. Findings from this study expanded on findings from earlier studies to affect the current practice of empiric therapy of CA-UTI in Uganda.Furthermore, an understanding of the most common host-associated factors to CA-UTIs in Uganda can help in developing preventive interventions against CA-UTIs, thus reducing on the disease burden and health costs.

\section{Methods}

\section{Study design and setting}

This was a cross-sectional study that was conducted in adults who presented with symptoms of a UTI at Mulago Hospital, assessment center which is the out-patient set- ting of Mulago National Referral Hospital. Patients were recruited from Mulago National Referral Hospital, assessment center, located about $200 \mathrm{~m}$ from the Makerere Medical Microbiology Laboratory. The Mulago Hospital assessment centre receives an average of 300 patients a day, some come in as self referrals and others are referrals from other hospitals and nearby medical centres. The urine samples were processed at the Makerere Medical Microbiology laboratory at the College of Health Sciences. This is a clinical microbiology laboratory that adheres to Good Clinical Laboratory Practices (GCLP).

\section{Sample size and study participants}

One hundred thirty nine patients were recruited for the study. The sample size of 139 was calculated using survey formula by Kish Leslie (1965); $\mathrm{n}=\mathrm{z}^{2} \mathrm{p}(1-\mathrm{p}) / \mathrm{d}^{2}$. Where $\mathrm{N}=$ estimated sample size, $\mathrm{Z}=\mathrm{Z}$ score for $95 \%$ confidence interval $=1.96, \mathrm{p}=$ disease prevalence; According to Mwaka et al, the prevalence of UTI is $10 \% 10$. , $d=$ acceptable margin of error $=5 \%=0.05$.

To estimate the sample size for the factors associated with UTIs, the method for calculating sample size for two proportions was employed. $\mathrm{n}=(\mathrm{Z} 1+\mathrm{Z} 2) 22 \mathrm{P}(1-\mathrm{P}) /(\mathrm{P} 2-$ P1)2where; $Z 1$ is $Z$ value at $95 \%$ level of significance $=$ $1.96, Z 2$ is $Z$ value at $80 \%$ power $=0.84, \mathrm{P} 1$ is proportion of patient with UTI among males $=14.1 \% 11$, P2 is proportion of patient with UTI among females $=27.1 \% 11$, $\mathrm{P}=(\mathrm{P} 1+\mathrm{P} 2) / 2$. The sample size calculated was less than the sample size for the prevalence so we used the bigger of the two sample sizes.

The study included individuals above 18 yrs of age who presented to Mulago hospital Assessment Center with symptoms suggestive of UTI including; lower abdominal or flank pain, dysuria and hematuria, urgency, frequency, hesitance, and consented to be part of the study. Patients who were mentally handicapped were not included in the study because they were unable to consent

\section{Study variables}

An interviewer administered questionnaire was used to collect information from the study participants as regards, demographic data: age, sex, Occupation, address, marital status and level of education. Symptoms of UTI and for how long they have been experiencing the symptoms. Host associated factors ,medical history including: antibiotics for current UTI episode, antibiotics in the past 
one month for any other illnesses, vaginal infections in females and treatment taken, chronic illnesses, history of UTI by the participant and any family members with a UTI. Sexual habits including, number of sexual partners, and number of times they have sex in a week, condom use and history of douching in female participants. Environmental hygiene including,if they have access to a personal toilet/latrine at home, use of public toilets/latrines in the past 6 months, and whether the toilets were for seating or squatting, the average number of times they use the toilets/latrines and whether they wash hands after visiting the toilets/latrines.

\section{Data and sample collection}

The staff members at Mulago hospital assessment center were briefed about the study and then qualified medical officers at the study site screened and diagnosed patients who presented with symptoms of UTI. Upon completion of the management plan, the medical officers would direct the patients to the study investigators at the study site. The study investigators would then explain the goal of the study to each patient in details and ask them for their consent for participation in the study. Individuals who consented were asked to fill in an interviewer administered questionnaire which was directed at demographic information, symptoms of UTI and host-associated factors for CA-UTI.

We collected 139 midstream urine samples from 139 study participants using a standard procedure and in a sterile, wide mouthed, screw-capped, transparent plastic container. The procedure was explained to each participant both males and females. For male patients they were given four pieces of sterile gauze, they were instructed to start by cleaning the tip of the penis with the two gauzes soaked in soap, then clean with the gauze soaked in normal saline and finally with the dry gauze. For the males who were not circumcised they were requested to draw back the foreskin before cleaning. The patients were requested to discard the few initial mls of urine and collect about $10-20 \mathrm{mls}$ of the mid-portion into the container provided. For females they were also given four pieces of gauze and were instructed to squat and part the labia then clean around the urethral opening from front to back starting with the two pieces of gauze that were soaked in soap, then clean with the gauze soaked in normal saline and dry with the dry gauze. . The patients were requested to discard the few initial mls of urine and collect about $10-20 \mathrm{mls}$ of the mid-portion into the container provided.

All MSU samples were packed in a cool box and transported to the Makerere Medical School Microbiology laboratory under 2 hours of collection. The Makerere Medical School Microbiology laboratory is about 200 meters away from Mulago Hospital Assessment Center. The samples were analyzed immediately after they had been entered in a log book on arrival at the laboratory. Macroscopically; the colour of urine was determined by visual inspection if colorless, pale yellow or blood stained. The consistency of urine was determined if clear or turbid. Microscopically; about $10 \mathrm{ml}$ of urine was centrifuged $500-1000 \mathrm{~g}$ for 5 minutes. The supernatant was discarded and the sediment was examined initially under X10 followed by X40 for White Blood Cells, Red Blood Cells, bacteria, yeasts, cell casts,Trichomonas vaginalis, motile trophozoites, Schistosoma haematobium eggs and bacteria $^{10}$. Chemical analysis; A urine dipstick was used to determine the following parameters; $\mathrm{pH}$, leukocyte esterase, glucose, bilirubin, urobilinogen, blood, ketones, nitrite production, and proteins in urine

\section{Culture}

Using a calibrated loop, $1 \mathrm{ml}$ of the MSU was inoculated onto MacConkey agar with crystal violet and 5\% sheep blood agar and Cystine Lactose Electrolyte Deficient (CLED) agar. CLED was used as it gives consistent results and allows the growth of both gram negative and gram positive bacterial pathogens, and it also prevents the swarming of Proteus species ${ }^{10}$.The agar plates that were streaked with the urine sample were incubated under aerobic conditions, at a temperature of $37^{\circ} \mathrm{C}$ and observed after 18-24 hours. Agar plates with pure growth and colonies were used for identification and sensitivity testing. The organisms were identified using biochemical tests including, urease, and motility tests, including triple sugar iron agar, Simmon's citrate agar, and lysine decarboxylase ${ }^{10}$.

\section{Sensitivity}

Sensitivity tests were done using the Mueller-Hinton-2agar, following the commercial disc diffusion techniques of Kirby-Bauer, against Ampicillin (10 mcg), Sulfamethoxazole-trimethoprim ( $25 \mathrm{mcg}$ and $125 \mathrm{mcg}$ ), gentamycin (10 mcg), ciprofloxacin (5mcg), Nitrofurantoin 
(300 mcg), cefuroxime (30 mcg), nalidixic acid (30 mcg), amoxicillin-clavulanic acid (20/10 mcg)12. Colony-saline mixtures were made my emulsifying colonies into sterile saline in test tubes and this colony-saline mixture were applied onto the surface of the Mueller-Hinton-2-agar.Antibiotic impregnated discs for the antibiotics mentioned above were placed on the agar surface with a minimum distance of $25 \mathrm{~mm}$ apart and the agar plates were incubated aerobically at a temperature of 370C for 18-24 hours. The zones of inhibition diameters were measured using a ruler and were compared against the zone diameter interpretative standards recommended by the National Committee for Clinical Laboratory Standards (NCCLS/ CLSI), 200513. The results after processing were recorded as sensitive or resistant to the antibiotic used.

\section{Data management and analysis}

All the raw data from questionnaires and microbiology analysis was recorded into a database generated using Epidata version 3.1 and then transferred to STATA version 12 for analysis. Numeric data was summarized using measures of central tendency while the categorical data was summarized using proportions and percentages. Comparisons were made between participants who had UTI and those who had no UTI. For the parametric continuous data, the comparisons were made using the student t-test while for the non parametric data; Wilcoxon Rank sum test was used. For the categorical data, comparisons were done using the Chi-squares and the Fisher's exact test. The outcome was dichotomized as presence or absence of UTI and logistic regression was used to assess for the associations. Bivariate analysis was applied and all the variables with a $\mathrm{p}$ value of 0.2 or less were entered into multivariate models. Interaction and confounding were assessed. All significance was put at $\mathrm{p}$ value of 0.05 or less.

\section{Ethical consideration}

We obtained approval for the study from School of Biomedical Sciences Research and Ethics Committee (SBSREC), Makerere University, College of Health Sciences. Approval was also obtained from Mulago Hospital Research and Ethics Committee, and written informed consents were obtained from every study participants. Finally, Laboratory IDs were assigned to patients' samples before analysis for confidentiality.

\section{Results}

\section{Socio-demographic and clinical characteristics}

A total of 139 participants took part in the study, of these $110(79.71 \%)$ were females, $71(52.21 \%)$ were married, $53(40.14 \%)$ had attained a secondary level of education and the great majority, 112( 81.75\%) lived within Kampala, as showed by the table of socio-demographics ( table 1).

Of all the study participants, $8(7.55 \%)$ practiced douch-

Table 1: Socio-demographic characteristics of patients with symptoms suggestive of UTI.

\begin{tabular}{llll}
\hline Variables & Categories & Number (n=138) & Percentages. \% \\
\hline Sex & Female & 110 & 79.71 \\
Address & Male & 28 & 20.29 \\
& Kampala & 112 & 81.75 \\
Wakiso & 12 & 8.76 \\
& Others* & 13 & 9.49 \\
& Student & 20 & 15.15 \\
Marital status & Self employed & 70 & 53.03 \\
& Civil servant & 7 & 5.30 \\
Notion & Not employed & 35 & 26.52 \\
& Single & 63 & 46.32 \\
& Married & 71 & 52.21 \\
& Separated & 2 & 1.47 \\
& Primary & 51 & 38.64 \\
& Secondary & 53 & 40.15 \\
& Tertiary & 25 & 18.94 \\
& No formal education & 3 & 2.27 \\
\hline
\end{tabular}


ing,11(11.11\%) had multiple sexual partners, only 16(16.16\%) used condoms, $116(84.06 \%)$ were using public toilets and majority $133(97.03 \%)$ reported to be wash- ing hands after using toilets, as shown in the table of risk factors for community acquired UTIs ( table 2)

Table 2: Risk factors for community acquired UTIs and clinical characteristics of the study participants.

\begin{tabular}{|c|c|c|c|c|c|}
\hline Variables & Categories & Number & Percentages. & & \\
\hline \multirow{2}{*}{$\begin{array}{l}\text { History of vaginal } \\
\text { infections }\end{array}$} & $33(50.00)$ & $33(50.00)$ & 0.015 & & \\
\hline & 26 and above & $50(70.42)$ & $21(29.58)$ & & \\
\hline \multirow[t]{2}{*}{$\operatorname{sex}$} & Female & $61(55.96)$ & $48(44.04)$ & 0.029 & \\
\hline & Male & $22(78.57)$ & $6(21.43)$ & & \\
\hline $\begin{array}{l}\text { Vaginal } \\
\text { infection in last } \\
4 \text { weeks. }\end{array}$ & Yes & $22(47.83)$ & $24(52.17)$ & & \\
\hline Douche & Yes & $4(50.00)$ & $4(50.00)$ & 0.713 & \\
\hline \multirow[t]{3}{*}{ Type of toilet } & Seater & $12(66.67)$ & $6(33.33)$ & & \\
\hline & Squatter & $48(64.00)$ & $27(36.00)$ & 0.442 & \\
\hline & Latrine & $11(50.00)$ & $11(50.00)$ & & \\
\hline Hand washing & Yes & $80(60.15)$ & $53(39.85)$ & & \\
\hline Amikacin & & -- & -- & -- & $1 / 1(100$ \\
\hline Vancomycin & & -- & -- & -- & -- \\
\hline Erythromycin & & -- & $1 / 3(33.3)$ & -- & -- \\
\hline Ceftizoxime & & $1 / 14(7.1)$ & -- & -- & -- \\
\hline Ceftazidine & & $1 / 14(7.1)$ & -- & -- & -- \\
\hline
\end{tabular}

*"-_“means there was missing information in that section.

Of the participants with symptoms suggestive of UTIs, 84 patient urine samples had no bacterial growth, $54(39.13 \%)$ were found to actually have UTIs, with 26(18.84\%) patient urine samples having pure bacterial growth while the remaining $28(20.29 \%)$ urine samples had mixed bacterial growth, one sample was contaminated and thus was not considered. Age and sex were significantly associated with UTIs with p- values of 0.015 and 0.029 respectively, as shown in the table for comparison between participants with UTI and no UTI (table 3) Macroscopy, Microscopy, culture and sensitivity 
Table 3: Comparison between participants with UTI and those with no UTI.

\begin{tabular}{|c|c|c|c|c|}
\hline & & No UTI ( 83 ) & UTI ( 54) & \\
\hline Variable & Categories & $\mathrm{N}($ percentage $)$ & $\mathrm{N}($ percentage $)$ & P-value \\
\hline Age & Below 26 & & & \\
\hline \multirow[t]{4}{*}{ Habit } & Not regular & $20(51.28)$ & $19(48.72)$ & \\
\hline & 1 to 2 & $17(80.95)$ & $4(19.05)$ & 0.114 \\
\hline & 3 to 4 & $13(56.52)$ & $10(43.48)$ & \\
\hline & Over 4 & $3(42.86)$ & $4(57.14)$ & \\
\hline Condoms & Yes & $9(56.25)$ & $7(43.75)$ & 0.724 \\
\hline Family history & Yes & $14(77.78)$ & $4(22.22)$ & 0.112 \\
\hline Personal toilet & Yes & $49(59.76)$ & $33(40.24)$ & 0.708 \\
\hline Public toilet & Yes & $71(61.74)$ & $44(38.26)$ & 0.527 \\
\hline \multirow[t]{3}{*}{ Type of toilet } & Seater & $12(66.67)$ & $6(33.33)$ & \\
\hline & Squatter & $48(64.00)$ & $27(36.00)$ & 0.442 \\
\hline & Latrine & $11(50.00)$ & $11(50.00)$ & \\
\hline Hand washing & Yes & $80(60.15)$ & $53(39.85)$ & \\
\hline
\end{tabular}

Most of the urine samples collected were pale yellow and clear 48(34.78\%),136 (97.85\%) had absent glucose and the mean ph was 6.5 with a standard deviation of \pm 1.43 , microscopically, $75(59.52 \%)$ of the urine samples had white blood cells,24(28.24\%) red blood cells and $10(21.74 \%)$ had casts,as showed in( table 4).

The commonest uropathogen isolated was Escherichia 
Table 4: Macroscopic and microscopic characteristics of the urine samples.

Except where otherwise stated the value is number (percentages)

\begin{tabular}{|c|c|c|}
\hline Variables & Categories & Value \\
\hline \multirow[t]{11}{*}{ Appearance } & Pale yellow, clear & $48(34.78)$ \\
\hline & Pale yellow, turbid & $43(31.16)$ \\
\hline & Yellow, clear & $9(6.52)$ \\
\hline & Yellow, turbid & $22(15.94)$ \\
\hline & Colourless, clear & $2(1.45)$ \\
\hline & Reddish, turbid & $4(2.90)$ \\
\hline & Amber, turbid & $3(2.17)$ \\
\hline & Dark yellow, clear & $2(1.45)$ \\
\hline & Dark yellow, cloudy & $1(0.72)$ \\
\hline & Blood stained & $1(0.72)$ \\
\hline & Others & $3(2.17)$ \\
\hline \multirow{2}{*}{ Glucose } & Present & $3(2.15)$ \\
\hline & Absent & $136(97.85)$ \\
\hline \multirow[t]{2}{*}{ Protein } & Present & $22(15.83)$ \\
\hline & Absent & $117(84.17)$ \\
\hline \multirow[t]{3}{*}{ Ketones } & + & $3(2.16)$ \\
\hline & ++ & $12(8.63)$ \\
\hline & Absent & $124(89.21)$ \\
\hline \multirow[t]{4}{*}{ Bilirubin } & + & $3(2.16)$ \\
\hline & ++ & $1(0.72)$ \\
\hline & Urobilinogen $2 \mathrm{mg} / \mathrm{dl}$ & $8(5.76)$ \\
\hline & absent & $127(93.52)$ \\
\hline$P h($ mean $) \pm S D$ & & $6.5 \pm 1.43$ \\
\hline \multirow{6}{*}{ Wbc1 - in chemistry } & + & $9(6.48)$ \\
\hline & ++ & $13(9.35)$ \\
\hline & +++ & $4(2.880$ \\
\hline & $25 / \mathrm{mcl}$ & $2(1.44)$ \\
\hline & $75 / \mathrm{mcl}$ & $3(2.16)$ \\
\hline & Absent & $108(77.70)$ \\
\hline \multirow[t]{5}{*}{ Rbc1- in chemistry } & + & $1(0.72)$ \\
\hline & ++ & $5(3.60)$ \\
\hline & +++ & $1(0.72)$ \\
\hline & $250 / \mathrm{mcl}$ & $1(0.72)$ \\
\hline & absent & $131(94.24)$ \\
\hline specific gravity(mean) \pm SD & & $1.005 \pm 0.009$ \\
\hline Nit- nitrates & + & \\
\hline \multirow{6}{*}{ Microscopy } & White blood cells & $75(59.52)$ \\
\hline & Red blood cells & $24(28.24)$ \\
\hline & casts & $10(21.74)$ \\
\hline & Epithelial cells & $24(55.81)$ \\
\hline & Yeast cells & $4(9.52)$ \\
\hline & Parasites & $8(100)$ \\
\hline
\end{tabular}

coli at $50 \%$, this was followed by Staphylococcus aureus with $15.4 \%$. Other organisms cultured included, Actinomyces israelli 1(3.8\%), Providentia spp 1(3.8\%), Citrobacter spp 1(3.8\%), Klebsiella spp 1(3.8\%), streptococcus 1(3.8\%) and others $4(15.4 \%)$. The sensitivity of Escherichia coli to qm- picillin and nitrofurantoin were $78.6 \%, 64.3 \%$ respectively, the sensitivity of Staphylococcus aureus to ciprofloxacin, nitrofurantoin and gentamycin were 100\%, 66.7\% and $66.7 \%$ respectively (table 5).

Bivariate 
Table 5: Antibiotic sensitivity patterns of the organisms isolated in the pure bacterial growths.

\section{Sensitivity results of some of the isolated uropathogens.}

\begin{tabular}{lclll}
\hline \multicolumn{5}{c}{ Sensitivity results of some of the isolated uropathogens. } \\
\hline Drug & $\begin{array}{l}\text { Escherichia } \\
\text { coli }\end{array}$ & $\begin{array}{l}\text { Staphylococcus } \\
\text { aureus }\end{array}$ & $\begin{array}{l}\text { Klebsiella } \\
\text { pneumoniae }\end{array}$ & $\begin{array}{l}\text { Providencia } \\
\text { spp }\end{array}$ \\
Nitrofurantoin & $9 / 14(64.3)$ & $2 / 3(66.7)$ & -- & $1 / 1(100)$ \\
Ciprofloxacin & $8 / 14(57.1)$ & $3 / 3(100)$ & -- & -- \\
Nalidixic acid & $2 / 14(14.3)$ & -- & -- & -- \\
Gentamycin & $8 / 14(57.1)$ & $2 / 3(66.7)$ & $1 / 1(100)$ & -- \\
Cotrimoxazole & -- & $2 / 3(66.7)$ & -- & -- \\
Augmentin & $5 / 14(35.7)$ & -- & -- & -- \\
Cefuroxime & $8 / 14(57.1)$ & -- & $1 / 1(100)$ & -- \\
Ceftriaxone & $6 / 14(42.9)$ & -- & -- & -- \\
Tetracycline & -- & $2 / 3(66.7)$ & -- & - \\
Oxacillin & -- & $1 / 3(33.3)$ & -- & -- \\
Chloramphenicol & $7 / 14(50)$ & $1 / 3(33.3)$ & $1 / 1(100)$ & -- \\
Ampicillin & $11 / 14(78.6)$ & -- & -- & $1 / 1(100)$ \\
Vancomycin & -- & -- & -- & -- \\
Erythromycin & -- & $1 / 3(33.3)$ & -- & -- \\
Ceftizoxime & $1 / 14(7.1)$ & -- & -- & -- \\
Ceftazidine & $1 / 14(7.1)$ & -- & -- & -- \\
\hline
\end{tabular}

*"--“means there was missing information in that section.

When the study participants were compared in a Bivariate analysis, age 26 and above (a OR=2.38; 95\%CI: 1.184.80), female sex (a OR=2.88; 95\%CI: 1.08-7.67) and being married (a OR=0.45; 95\%CI: 0.22-0.92) were significantly associated with UTIs, (table 6 )

Multivariate
When the study participants were compared in a multivariate logistic analysis, age 26 and above $(\mathrm{a} \mathrm{OR}=2.59$; 95\%CI: 1.12-5.99), female sex (a OR $=3.33$; 95\% CI: 1.11-9.95) and being married (a OR=0.29; 95\%:0.130.67) were significantly associated with UTIs, (table 6)

\section{Discussion}


Table 6: Bivariate and multivariate analysis of the risk

factors for community acquired UTIs

\begin{tabular}{|c|c|c|c|c|c|}
\hline Variable & Categories & $\begin{array}{l}\text { Unadjusted OR ( } \\
95 \% \mathrm{CL})\end{array}$ & P-value & $\begin{array}{l}\text { Adjusted } \\
\text { OR(95\% CL) }\end{array}$ & p-value \\
\hline \multirow[t]{2}{*}{ Age } & Below 26 & 1 & & 1 & \\
\hline & 26 and above & $2.38(1.18-4.80)$ & 0.015 & $\begin{array}{l}2.59(1.12- \\
5.99)\end{array}$ & $\mathbf{0 . 0 2 5}$ \\
\hline \multirow[t]{2}{*}{$\operatorname{sex}$} & Male & 1 & & 1 & \\
\hline & Female & $2.88(1.08-7.67)$ & 0.034 & $\begin{array}{l}3.33(1.11- \\
9.95)\end{array}$ & $\mathbf{0 . 0 3 1}$ \\
\hline \multirow[t]{2}{*}{ Practice Douche } & Yes & 1 & & & \\
\hline & No & $0.76(0.18-3.23)$ & 0.714 & & \\
\hline \multirow[t]{4}{*}{ occupation } & Student & 1 & & & \\
\hline & Self employed & $0.53(0.17-1.62)$ & 0.268 & & \\
\hline & Civil servant & 1 & & & \\
\hline & Not employed & $0.37(0.11-1.26)$ & 0.114 & & \\
\hline \multirow{2}{*}{ Marital status } & Single & 1 & & 1 & \\
\hline & Married & $0.45(0.22-0.92)$ & 0.030 & $\begin{array}{l}0.29(0.13- \\
0.67)\end{array}$ & 0.004 \\
\hline \multirow[t]{2}{*}{ More than 1 sexual partner. } & Yes & 1 & & & \\
\hline & No & $2.90(0.58-14.46)$ & 0.194 & & \\
\hline \multirow{2}{*}{ Condom use } & Yes & 1 & & & \\
\hline & No & $0.82(0.27-2.42)$ & 0.724 & & \\
\hline \multirow[t]{3}{*}{ Level of education } & None & 1 & & & \\
\hline & $\begin{array}{l}\text { Primary } \\
\text { Secondary }\end{array}$ & $3.1(0.26-36.48)$ & 0.368 & & \\
\hline & $\begin{array}{l}\text { Secondary } \\
\text { Tertiary }\end{array}$ & $3.55(0.28-44.88)$ & $\begin{array}{l}0.423 \\
0.327\end{array}$ & & \\
\hline \multirow[t]{2}{*}{ Antibiotics for current Uti } & Yes & 1 & & & \\
\hline & No & $1.61(0.75-3.44)$ & 0.212 & & \\
\hline \multirow[t]{2}{*}{ Antibiotic-use in last 30 days } & Yes & 1 & & & \\
\hline & No & $1.25(0.58-2.70)$ & 0.565 & & \\
\hline \multirow[t]{2}{*}{ Any chronic illnesses } & Yes & 1 & & 1 & \\
\hline & No & $3.16(0.65-15.24)$ & 0.151 & $\begin{array}{l}2.61(0.46- \\
14.69)\end{array}$ & 0.275 \\
\hline \multirow[t]{2}{*}{ Previous history of UTI. } & Yes & 1 & & & \\
\hline & No & $0.99(0.47-2.07))$ & 0.985 & & \\
\hline \multirow{2}{*}{ Family member with UTI } & Yes & 1 & & 1 & \\
\hline & No & $2.52(0.78-8.12)$ & 0.121 & $\begin{array}{l}3.56(0.99- \\
12.80)\end{array}$ & 0.052 \\
\hline \multirow{2}{*}{ Personal toilet/latrine. } & Yes & 1 & & & \\
\hline & No & $0.87(0.43-1.77)$ & 0.708 & & \\
\hline \multirow[t]{2}{*}{ Use of public toilet } & Yes & 1 & & & \\
\hline & No & $1.34(0.53-3.37)$ & 0.528 & & \\
\hline \multirow{2}{*}{ Hand washing after toilet } & Always & 1 & & & \\
\hline & Not always & $1.98(0.33-11.80)$ & 0.450 & & \\
\hline \multirow[t]{3}{*}{ Type of toilet. } & Seater & 1 & & & \\
\hline & Squatter & $1.955(0.691-5.534)$ & 0.206 & & \\
\hline & Latrine & $1.667(0.387-7.170)$ & 0.493 & & \\
\hline
\end{tabular}

This study looked at the factors associated with community acquired urinary tract infections, the common uropathogens isolated and their drug sensitivity patterns. The study revealed that age 26 years and above, female sex and marital status of the participants were significantly associated with CA-UTIs. The commonest uropathogens isolated were Escherichia coli (50\%), and Staphylococcus aureus $(15.4 \%)$. There was high resistance to the common prescribed antibiotics for CA-UTIs in Uganda such as erythromycin, cotrimoxazole and nalidixic acid with most uropathogens showing greater than $50 \%$ resistance rates. From our study, 54(39.13\%) urine samples were found to actually have UTIs, with $26(18.84 \%)$ patient urine sam- ples having pure bacterial growth while the remaining $28(20.29 \%)$ urine samples had mixed bacterial growth. In the study by Mwaka et al the prevalence of significant bacteriuria among non pregnant women attending the same study setting was $10 \%{ }^{8}$. The study by Andabati et al among antenatal mothers in Mulago Hospital, Uganda found a $13.3 \%$ prevalence of asymptomatic bacteriuria ${ }^{14}$. These finds are much comparable to the findings from our study. Another study from Ethiopia by Moges et al ${ }^{15}$, found a prevalence of UTI of $39.5 \%$, which is about 2 times what we found. The study by Moges et al included in-patients which could account in part for the difference in prevalence of UTI.

The findings of age, female sex and marital status as 
factors significantly associated with CA-UTIs have been reported in other studies. ${ }^{7,16}$. There are no studies involving both men and women determining factors associated with community acquired UTIs have been cited in Uganda. Urinary tract infections due to E.Coli are a common finding in women and this is associated with the close proximity of the female urethral meatus and the anal orifice.

The most frequent isolated uropathogen from our study was Escherichia coli, accounting for $50 \%$ of all the isolates. This is much comparable to earlier studies in Africa and also Uganda where E.coli was isolated as the most frequent uropathogen. Mwaka et al isolated E.coli at $57.5 \%$ in his study though his study was only limited to nonpregnant women and no studies involving both men and women have been cited in our setting and other studies have shown similar findings of E.coli as the most frequent uropathogen in CA-UTIs ${ }^{15,17,18}$.

The second most frequent bacteria isolated were Staphylococcus aureus at isolation rates of $15.4 \%$, earlier studies in Mulago hospital had also isolated S.aureus as a uropathogen ${ }^{8,17,19}$, though most of these studies were limited to women in the reproductive ages, men and elderly women were not part of these studies. This finding is however different from a recent study by Odongo etal in Gulu referral hospital that found Staphylococcal species as the most frequent uropathogens in their study at $46.3 \%{ }^{20}$. The other uropathogens isolated included Klebsiella pneumonia, Providencia specie, Citrobacter specie, Actinomyces israelli.

Escherichia coli which was the commonest uropathogen isolated showed generally high sensitivity to Ampicillin, and Nitrofurantoin at 78.6\%,64.3\% respectively and reduced sensitivity to ciprofloxacin at $57.1 \%$. An earlier study in the same study setting by Mwaka et al involving non pregnant women had shown high sensitivity of E.Coli to Nitrofurantoin at $100 \%$ sensitivity rates, this shows a decline in the sensitivity rates to this antibiotic by E.coli in the same study setting and comparable sensitivity patterns had been shown by other studies in other settings in Africa ${ }^{15,17,19,21}$. Also a recent study in Gulu Regional Hospital in northern Uganda by Odongo et al has shown high resistance to Nitrofurantoin which is a rather worrying trend.

Staphylococcus aureus the second most common uropatho- gen isolated showed high sensitivity to ciprofloxacin, Nitrofurantoin and gentamycin at 100\%, 66.7\% and 66.7\% respectively. The sensitivity of S.aureus to ciprofloxacin in our study was $100 \%$ which is higher than the other similar studies in the region ${ }^{8,20}$. We can attribute this to the few growths of S.aureus that were set for ciprofloxacin in our study. The study by Mwaka et al had also showed high sensitivities of S.aureus to the antibibiotics with Nitrofurantoin,ciprofloxacin and gentamycin showing sensitivities of $100 \%, 68.4 \%$ and $68.4 \%$ respectively ${ }^{8}$, though this study was only in non pregnant women. The study by Odongo et al in Gulu regional hospital in Northern Uganda also showed high sensitivities to gentamycin at $85.4 \%{ }^{20}$. Most organisms were more than $50 \%$ sensitive to nitrofurantoin and gentamycin.

Most organisms had low sensitivity to antibiotics such as cotrimoxazole, nalidixic acid and erythromycin. In the Uganda clinical guidelines, cotrimoxazole is one of the recommended first line antibiotics for empirical management of UTIs, but our study showed a high resistance of the common uropathogens to cotrimoxazole. The high resistance to cotrimoxazole may be explained by factors such as the use of cotrimoxazole as prophylaxis against opportunistic infections among HIV positive patients ${ }^{22}$ and the use of sulfadoxine-pyrimethamine which shares enzyme targets with cotrimoxazole as routine prophylaxis during pregnancy ${ }^{22}$.

Of all the urine samples collected, there were 26 pure cultural growths which was small given the fact that all the patients had reported symptoms suggestive of UTI. The small figures of pure cultural growths could be explained by factors such as the use of over the counter antibiotics by the patients either for the current UTI episodes or other illnesses before coming to hospital which affects the culture results.

\section{Conclusion}

There are known factors associated with CA-UTIs such as age, female sex and marital status. Escherichia coli and Staphylococcus aureus are major causes of CA-UTIs in the study area according to our findings. There was generally high sensitivity to nitrofurantoin and gentamycin by most of the uropathogens isolated. There were high resistance rates to cotrimoxazole and erythromycin drugs that are mainly used as first line in the empirical management of 
community acquired urinary tract infections according to the Uganda clinical guidelines.

\section{Limitations}

Many of the patients most likely have had some form of empirical antibiotic treatment prior to coming to Mulago since the hospital is a National Referral centre. As a result of this undocumented prior antibiotic treatment, the true catch rate of the organisms in the urine could have been obscured. However we tried to limit this by verbally ascertaining whether the patient had recently been treated for a UTI.

For many of the variables, we relied on self report of the patient. This is subject to several forms of bias including recall bias, social desirability bias among others. With the limited resources at our disposal we could not utilize anymore sensitive tests for several of the variables

\section{Recommendations}

Owing to the high resistance rates to the recommended first line antibiotics in the Uganda clinical guidelines, there is need for bigger studies that can be used to effect changes in the guidelines for the management of community acquired UTIs in order to suite the changing resistance patterns and also save the patients the complications that come with these infections when mismanaged.

\section{Abbreviations}

UTI,Urinary tract infections; CA-UTI,Community acquired urinary tract infection;HCWs, health care workers; GCLP,Good Clinical Laboratory Practices(GCLP);MSU,Mid-Stream Urine;CLED,Cystine Lactose Electrolyte Deficient; NCCLS,National Committee for Clinical Laboratory Standards; SBS-REC,Biomedical Sciences Research and Ethics Committee; OR,Odds Ratio; E.Coli,Escherichia Coli; spp,species.

\section{Competing interests}

The authors declare no competing interests.

\section{Authors' contributions}

$\mathrm{KD}$ participated in writing the proposal for the study, data and sample collection, data analysis and writing the manuscript. DDA participated in writing the manuscript, data and sample collection. KAG, NR. NS and KMR participated in writing the manuscript and data and sample collection. BA and FCN guided us in writing of the proposal and writing the manuscript. KS participated in data analysis and also guided in the writing of the manuscript.

\section{Acknowledgements}

We offer our thanks to all the participants who took part in this study. The project was supported by the MESAU-MEPI Programmatic Award through Award Number1R24TW008886 from the Fogarty International Center. The content is solely the responsibility of the authors and does not necessarily represent the official views of the Fogarty International Center or the National Institutes of Health. We are grateful to Makerere University College of health sciences.

\section{References}

1. Sabrina J: Antimicrobial resistance among producers and non-producers of extended spectrum beta-lactamases in urinary isolates at a tertiary Hospital in Tanzania. BMC Research Notes 2010:3:348.

2. Gonzalez C: Treatment of urinary tract infection: what's old, what's new, and what works. World J Urol 1999:17:372-382.

3. Nimri L: Community-Acquired Urinary Tract Infections in a Rural Area in Jordan: Predominant Uropathogens, And their Antimicrobial Resistance. WebmedCentral MICROBIOLOGY 2010:1(9):WMC00660

4. Bahadin J: Aetiology of community-acquired Urinary tract infection and antimicrobial susceptibility patterns of uropathogens isolated. Singapore Med J 2011:52(56): 415. 5. Hooton T: Diagnosis and treatment of uncomplicated urinary tract infection. Infect Dis Clin North Am 1997:11(13):551-581.

6. Guneysel O: Trimethoprim/sulfamethoxazole resistance in urinary tract infections. $J$ Emerg Med 2009:36(34):338-341.

7. Ronald A: The etiology of urinary tract infection: traditional and emerging pathogens. The American Journal Of Medicine 2002: July; 113(111): pp. 114-119.

8. Mwaka A: Bacteriuria among adult non-pregnant women attending Mulago hospital assessment centre in Uganda. Afri Health Sci 2011 11(12): 182-189.

9. Kyabbagu D: The sensitivities to the first antibiotic therapy of the common urinary tract bacterial infections detected in urine samples in a metropolitan hospital in Kampala(Ug). Afri Health Sci 2007:7(4): 214-222.

10. Cheesbrough M: District Laboratory Practice in Trop- 
ical Countries. Examination of urine and antimicrobial sensitivity testing. 2000:pp. 105-143. Part 102.

11. Mulugeta K: Prevalence and antibiogram of bacterial isolates from urinary tract infections at Desire Health Research Laboratory Ethiopia. Asian Pac J Trop Biomed 2014:4(2): 164-168.

12. BauerA: Antibiotic susceptibility testing by a standardized single disk method. Am J Clin Pathol 1966:45:493496.

13. Marie B: Manual of Antimicrobial Susceptibility Testing. 2005.

14. Andabati G: Microbial aetiology and sensitivity of asymptomatic bacteriuria among ante-natal mothers in Mulago hospital, Uganda. Afr Health Sci 2010 Dec; 10(4): 349-3522010.

15. Moges A: Antibiotic sensitivities of common bacterial pathogens in urinary tract infections in Gondar Hospital, Ethiopia,. East Afr Med J 2002:79; 140 - 142.

16. Astral Z: Relationship between demographic characteristics and community-acquired urinary tract infection. 2002. PubMed

17. Wanyama J: Prevalence, bacteriology and microbial sensitivity patterns among pregnant women with clinically diagnosed urinary tract infections in Mulago Hospital Labour Ward. MMed dissertation of Wanyama, Makerere University 2003.

18. Kayima J: Asymptomatic bacteriuria among diabetics attending Kenyatta National Hospital. East AfrMed J 1996:73: $524-526$.

19. Ouma J: Prevalence and antimicrobial sensitivityn of major bacteria associated with urinary tract infection among diabetic patients in Mulago Hospital. Special Project Report Makerere University 2001.

20. Odongo: Antibiograms from community - acquired uropathogens in Gulu,northern Uganda- a cross - sectional study. BMC Infectious Diseases 2013(13):193.

21. Masinde A: Prevalence of urinary tract infection among pregnant women at Bugando Medical Centre, Mwanza, Tanzania. Tanzania Journal of Health Research 2009:11:154 - 161.

22. Authority MoHaTUND: Ugaanda Clinical Guidelines. National Guidelines on Management of Common Disease Conditions. 2010(kisubi, Uganda: Marianum Press Ltd; 2010). 